\title{
Experimental Coxiella burnetii infection in pregnant goats: excretion routes
}

\author{
Nathalie Arricau BouverY*, Armel Souriau, Patrick LECHOPIER, \\ Annie RODOLAKIS
}

Pathologie Infectieuse et Immunologie, INRA, Tours-Nouzilly, 37380 Nouzilly, France

(Received 15 October 2002, accepted 21 March 2003)

\begin{abstract}
Q fever is a widespread zoonosis caused by Coxiella burnetii. Infected animals, shedding bacteria by different routes, constitute contamination sources for humans and the environment. To study Coxiella excretion, pregnant goats were inoculated by the subcutaneous route in a site localized just in front of the shoulder at 90 days of gestation with 3 doses of bacteria $\left(10^{8}, 10^{6}\right.$ or $10^{4}$ I.D. $)$. All the goats aborted whatever the dose used. Coxiella were found by PCR and immunofluorescence tests in all placentas and in several organs of at least one fetus per goat. At abortion, all the goats excreted bacteria in vaginal discharges up to 14 days and in milk samples up to 52 days. A few goats excreted Coxiella in their feces before abortion, and all goats, excreted bacteria in their feces after abortion. Antibody titers against Coxiella increased from 21 days post inoculation to the end of the experiment. For a $\mathrm{Q}$ fever diagnostic, detection by PCR and immunofluorescence tests of Coxiella in parturition products and vaginal secretions at abortion should be preferred to serological tests.
\end{abstract}

Coxiella burnetii / goat / experimental infection / abortion / shedding

\section{INTRODUCTION}

Coxiella burnetii is an obligate intracellular bacterium responsible for Q fever, a widespread zoonosis [14]. Infection in man and animals has been worldwide reported $[4,5]$ with the exception of New Zealand [18]. Human infection could be acute (pneumonia, hepatitis, influenza-like symptoms and headache), or chronic (endocarditis, granulomatous hepatitis and abortion). In animals, $C$. burnetii usually causes reproductive disorders (abortion, retained placenta, endometritis, infertility and low birth weight) that are responsible for important economic losses.

The source of human infection is often unidentified, although sheep and goats are more frequently involved in the disease cycle than other animal species. The main route of $C$. burnetii infection is by inhalation of contaminated aerosols containing the microorganism shed from infected animals [1, 25, 26, 28, 30, 31, 34, 35]. After becoming infected, female animals shed large quantities of Coxiella into the

\footnotetext{
* Correspondence and reprints

Tel.: (33) 2474276 34; fax: (33) 2474277 79; e-mail: Nathalie.Bouvery@ tours.inra.fr
} 
environment during abortion or normal delivery through birth fluids, placenta and fetal membranes [3, 6, 23, 24, 32]. Moreover, following parturition, these infected animals excrete the bacteria via the urine, feces, vaginal discharges and milk for several months [10, 17, 19, 20]. Nevertheless, the $C$. burnetii shedding kinetics is not well known. Oral transmission is less common, but the consumption of contaminated raw milk and dairy-products could be a source of infection $[8,12,16]$. Thus, most people could be contaminated by $C$. burnetii, but staffs working with ruminants appear to be the most exposed [17]. An improved knowledge of excretion routes of $C$. burnetii by domestic ruminants is required to reduce the risk of human and animal contaminations. Experimental models have already been performed on sheep or cattle $[7,22,34]$. Some discrepancies have been obtained between the results that may be explained by the different route, the infective dose and the $C$. burnetii strain used. No model is available yet to reproduce $\mathrm{Q}$ fever in goats. Moreover, very few data have been reported about Coxiella excretion by naturally infected goats. However, $\mathrm{Q}$ fever abortions in goat herds are more important than in sheep flocks since they could rise up to $90 \%$ in females [23], and goat cheese produced from raw milk is eaten in many countries. So, the setting up of an experimental infection of goats is necessary to better understand the routes and duration of Coxiella excretion.

\section{MATERIALS AND METHODS}

\subsection{Coxiella burnetii strain}

The Coxiella burnetii strain $\mathrm{CbC} 1$, used in the study, was originally isolated from the placenta of an aborted goat in a French caprine herd (Allier France). It was identified as a Coxiella species by phenotypic and genotypic characterization. This strain was isolated by intraperitoneal inoculation of $3 \mathrm{OF} 1$ mice ( 8 weeks old) with $0.2 \mathrm{~mL}$ of goat placenta homogenate. The mice were killed nine days post inoculation (p.i.) and their spleens were sampled and reinoculated to specific pathogen free embryonated hen eggs. C. burnetii $\mathrm{CbC} 1$ in its $3 \mathrm{rd}$ passage in the chicken embryo was aliquoted and frozen at $-70{ }^{\circ} \mathrm{C}$ before use as the challenge strain $\left(10^{9}\right.$ infective mouse doses (I.D.) $/ \mathrm{mL}$ ). The infective mouse dose was calculated by peritoneal inoculation of mice with decimal dilutions of C. burnetii $\mathrm{CbC} 1$ and detection of Coxiella in the spleen by PCR.

\subsection{Animals and experimental protocol}

The animal experimentation was carried out over a four-month period in a level 3 biosecurity experimental building reserved for trained staff only working in adequate conditions of protection. Nineteen oneyear-old and healthy pregnant goats, obtained from Chlamydia and Coxiella specific serologically negative herds with no history of abortion, were randomly allotted into three experimental groups. They were inoculated by the subcutaneous route in a site localized just in front of the shoulder at 90 days of gestation, with $10^{8}$ (6 goats, $10^{8}$ group), $10^{6}$ (6 goats, $10^{6}$ group) or $10^{4}$ I.D. of the $\mathrm{CbC} 1$ strain (7 goats, $10^{4}$ group). The animals were kept in separate rooms in a security building for about 6 weeks after delivery. The animals were observed daily for clinical signs. Rectal temperatures were measured, once a day, the day before and during 8 days p.i. At the end of the study, the animals were necropsied for a detection of C. burnetii in different organs.

\subsection{Diagnostic tests}

\subsubsection{Samples}

Blood samples were collected for serological tests at the time of inoculation and then fortnightly. Fecal samples were collected directly from the rectum 24 days 
after inoculation and then fortnightly. Placental cotyledons were collected at parturition. The spleen, liver, lung, stomach and peritoneal fluids were collected from aborted fetuses, still-borns or kids dying within $24 \mathrm{~h}$ after birth. Vaginal swabs were collected with dry sterile cotton swabs from each animal after parturition, on the 3 subsequent days and then every week. Milk samples were taken at the parturition day and daily for five days after, and then every week. Prescapular, mammary and head (submaxillary, parotid, retropharyngeal) lymph nodes, liver, spleen and udder were collected following necropsy of all infected animals at the end of the experiment. The samples were kept at $-80^{\circ} \mathrm{C}$ for further PCR analysis to detect C. burnetii.

\subsubsection{Serological tests}

The sera were tested for the presence of specific antibodies for $C$. burnetii with an ELISA assay (CHEKIT-Q-Fever enzyme immuno-assay kit; Bommeli diagnostics, Switzerland) which was carried out as recommended by the manufacturer, using microtiter plates precoated with a mix of purified C. burnetii phase I + II antigens. Positive and negative controls were included in each test. The sample OD were expressed as a percentage of the positive control OD value which was considered to be $100 \%$. Goat sera were considered positive if they had an OD percentage of 50\% or more, doubtful if the OD percentage was between 40 and $50 \%$, and negative if the OD percentage was less than $40 \%$.

\subsubsection{C. burnetii DNA purification from samples}

Feces and vaginal swab samples were prepared as previously described [10]. Placental cotyledons, organs and lymph nodes from goats and fetus organs were homogenized with a Stomacher (Seward Medical, London, UK) in sterile saline buffer. C. burnetii DNA was extracted from $50 \mu \mathrm{L}$ of stomach content, peritoneal fluid, organ and lymph node homogenates, or from $100 \mu \mathrm{L}$ of milk or feces with the QIAamp DNA mini kit (Qiagen), according to the manufacturer's instructions.

\subsubsection{PCR assay}

PCR assays were performed as previously described [9] with $2.5 \mu \mathrm{L}$ of DNA extract. The PCR reaction was carried out in an automated DNA thermal cycler (UNO Thermobloc; Biometra); PCR products $(687 \mathrm{bp} ; 10 \mu \mathrm{L})$ were analyzed by electrophoresis on $1 \%$ agarose gel, stained with ethidium bromide, visualized under an ultra-violet transilluminator and photographed. When no DNA was amplified, 1:10 and 1:100 DNA dilutions were made and new PCR were performed. If the PCR was positive at one of these dilutions, the sample was then considered positive.

\subsubsection{Immunofluorescence assay}

An indirect immunofluorescence (IFA) test was applied for $C$. burnetii detection in smears of placental cotyledon samples on 3-well or 18-well microscope slides (Poly Labo), as previously described [10] using a specific serum obtained from mice inoculated with the Nine Mile $C$. burnetii strain. The slides were examined with a $40 \times$ objective (400× magnification) under a fluorescence microscope (Leitz Aristoplan) for brilliant green staining of $C$. burnetii microorganisms. The number of $C$. burnetii was estimated in different microscopic fields and scored as either no organisms (-) or 10 or more C. burnetii specific views per field (+). Positive Coxiella and negative controls were included in each test run.

\subsection{Statistical analysis}

The results were compared by the Kruskal-Wallis test with StatXact 5 CYTEL Software Corporation. 


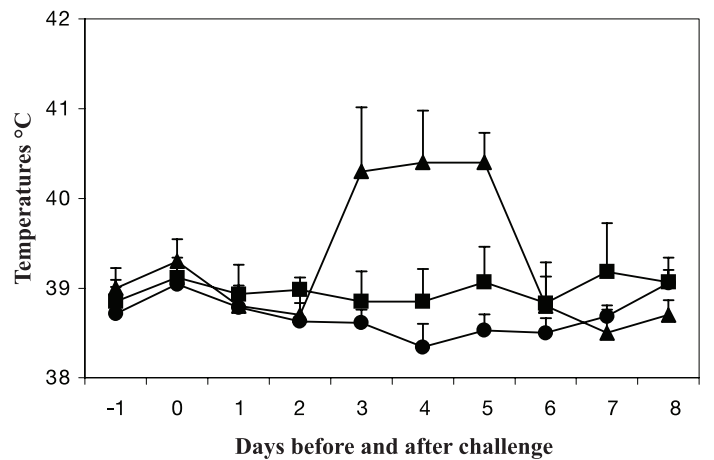

Figure 1. Mean changes in body temperature after the infectious challenge of 6 goats of the $10^{8}$ group ( $\triangle$ ), 6 goats of the $10^{6}$ group ( ) and 7 goats of the $10^{4}$ group $(\bullet)$. Rectal temperatures $\left({ }^{\circ} \mathrm{C}\right)$ were taken the day of pre-inoculation $(-1)$, the day of inoculation (0) and the following days (1 to 8).

\section{RESULTS}

\subsection{Clinical response after challenge}

A rise of rectal temperatures was only seen in the $10^{8}$ group; for the two other groups, as there was no control group, their rectal temperatures were not different to the ones before inoculation, except for two goats (Fig. 1). This fever began on the 3rd day after inoculation for all the goats of the $10^{8}$ group and lasted 3 to 5 days. An acute localized reaction also developed around the site of inoculation after 4 days p.i. for all the goats of this group, except for goat No. 132. Only 2 goats of the $10^{6}$ group presented a rise in rectal temperature, which began on the 5th and 7th days after challenge and lasted 3 and 1 days respectively; these 2 goats also showed an acute localized reaction after 5 days p.i. The rectal temperatures of the $10^{4}$ group goats were never over $39.5{ }^{\circ} \mathrm{C}$ until the 8 th day. No acute localized reaction was visible for the $10^{4}$ group goats.

\subsection{Antibody response}

According to the threshold of positivity given by the manufacturer of the kit, all goats became seropositive except the aborted goat No. 132 ( $10^{8}$ group) which had a doubtful serology throughout the experimentation. There was a seroconversion for all the infected goats which began around

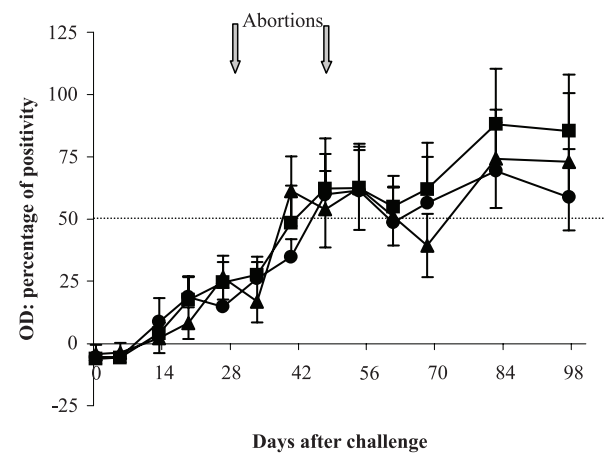

Figure 2. ELISA serological responses following Coxiella burnetii infection. According to the threshold of positivity given by the marker of the kit, the goats were considered to be positive if they had an OD percentage of $50 \%$ or more. The arrows represent the two abortion periods. Goat $10^{8}$ group (४); goat $10^{6}$ group $(\square)$; goat $10^{4}$ group $(\bullet)$.

21 days p.i. and gradually increased up to the end of the experiment (Fig. 2), but it was below the threshold of positivity of the kit the first weeks after inoculation up to 7 weeks for the majority of the goats of the three groups. According to the threshold of positivity of the kit, the first antibodies against phase I + II antigens of $C$. burnetii were detected 42 days after inoculation for $5 / 7$ of the $10^{4}$ group, $3 / 6$ of the $10^{6}$ group and $0 / 6$ goats of the $10^{8}$ group, and detection of antibodies was only positive for $3 / 7$ goats ( $10^{4}$ groups), $2 / 6$ goats 
Table I. Results of the abortion syndrome of goats inoculated with Coxiella burnetii and bacteriological investigations in placental cotyledons and fetuses by PCR.

\begin{tabular}{lccc}
\hline & \multicolumn{3}{c}{ Challenge dose } \\
\cline { 2 - 4 } & $10^{8}$ & $10^{6}$ & $10^{4}$ \\
\hline Goats & 6 & 6 & 7 \\
number of goats & $122 \pm 13$ & $130 \pm 10$ & $124 \pm 7$ \\
duration of gestation (days) $^{\mathrm{a}}$ & $1.7 \pm 0.8$ & $2.5 \pm 0.8$ & $2.6 \pm 0.8$ \\
number of fetus/goat & $6 / 6$ & $6 / 6$ & $7 / 7$ \\
Placenta (No. positive/total) & & & \\
Fetus (No. positive/total) & $3 / 7$ & $9 / 15$ & $14 / 16$ \\
Peritoneal content & $3 / 8$ & $4 / 13$ & $13 / 19$ \\
Stomach content & $6 / 9$ & $13 / 15$ & $11 / 19$ \\
Spleen & $7 / 9$ & $14 / 15$ & $12 / 19$ \\
Lung & $7 / 9$ & $15 / 15$ & $12 / 19$ \\
Liver & 62 & 75 & 67 \\
Total (\% positive) & & &
\end{tabular}

${ }^{a}$ Mean \pm SD.

b All placenta smears were also positive by immunofluorescence.

$\left(10^{6}\right.$ groups) and $1 / 6$ goats $\left(10^{8}\right.$ groups) at the day of abortion, but all the aborted goats became ELISA positive between 1 and 36 days after parturition (between days 40 and 82 p.i.).

\subsection{Abortion syndrome and fetal contamination (Tab. I)}

One goat (No. 132 of the $10^{8}$ group) aborted 12 days after the inoculation; all the other pregnant goats aborted between days 25 and 48 p.i., corresponding to days 115 and 138 of gestation. Whatever the dose used, none of the kids survived more than $24 \mathrm{~h}$. There were two abortion outbreaks, the first around 29 days p.i. and the second around 43 days p.i., with no significant difference between the duration of pregnancies between the 3 groups.

The cotyledons of all the aborted goats were positive for $C$. burnetii by immunofluorescence test and PCR. C. burnetii was detected in the majority of the aborted kids whatever the dose used. The most frequently contaminated samples were the liver (79\% positive) and lungs (77\% positive). There was no significant difference between the 3 groups.

\subsection{Excretion of C. burnetii}

\subsubsection{Fecal excretion}

C. burnetii was detected in the feces of all aborted goats, whatever the dose used (Fig. 3). The first detection happened 25 days p.i. in fecal samples of 6 goats, which excreted bacteria before the abortion. Moreover fecal excretion was discontinuous for $4 / 9$ goats of the $10^{6}$ group and $5 / 8$ goats of the $10^{4}$ group; negative results were obtained from some samples during the period of excretion. The analysis of the results with the Kruskal-Wallis test did not show a significant difference between the three groups. The mean duration of excretion (comprising continuous and discontinuous shedding) was 20 days.

\subsubsection{Vaginal excretion}

At the time of abortion and the 2 subsequent days, $C$. burnetii organisms were intensively shed in vaginal fluids of all aborted goats except for the goat No. 132 of the $10^{8}$ group which aborted 12 days p.i. (Fig. 4). This excretion decreased with time and Coxiella was not detected 3 days 

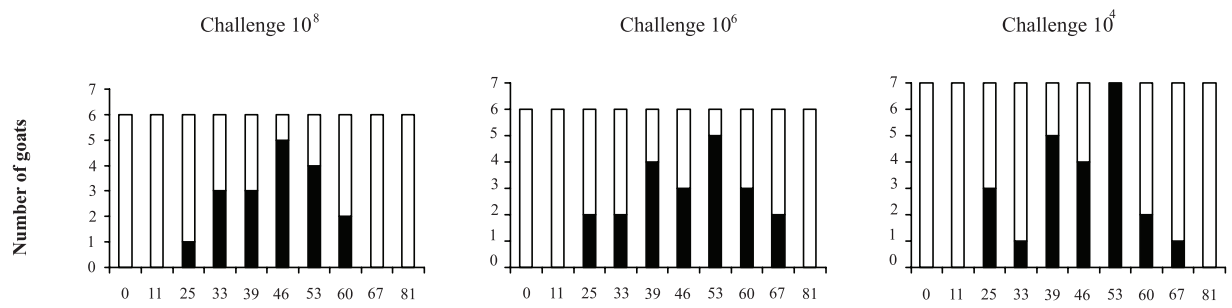

Days after inoculation

Figure 3. Assessment by PCR of the shedding of Coxiella burnetii in fecal samples the day of challenge and the following days. - positive PCR, $\square$ negative PCR.

Challenge $10^{8}$

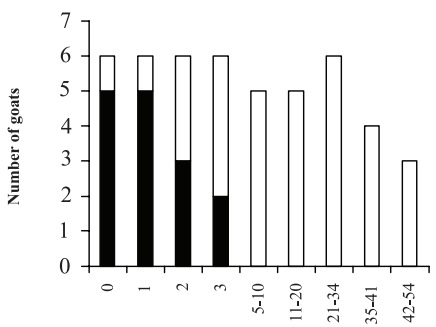

Challenge $10^{6}$

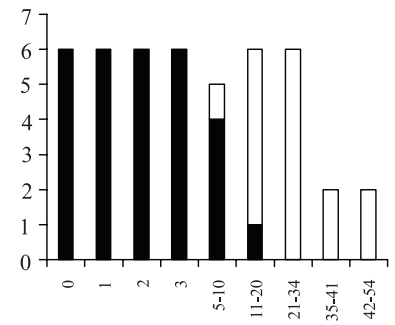

Days after abortion
Challenge $10^{4}$

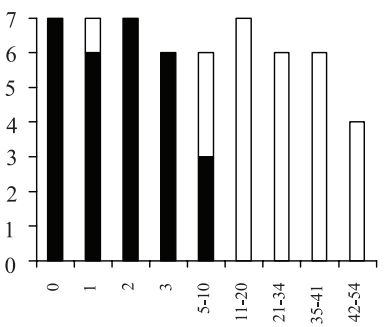

Figure 4. Assessment by PCR of the shedding of $C$. burnetii in vaginal swabs the day of abortion and the following days. a positive PCR, $\square$ negative PCR.

after abortion for the $10^{8}$ group, 10 days for the $10^{4}$ group and 14 days for the $10^{6}$ group. The mean duration of excretion was 2 days for the $10^{8}$ group, 8 days for the $10^{6}$ group and 4.5 days for the $10^{4}$ group. Analysis of these results with the KruskalWallis test showed only significant difference between the $10^{8}$ group and the $10^{6}$ group $(P=0.0085)$.

\subsubsection{Milk excretion}

C. burnetii was always excreted in milk after abortion and the following days, except for goat No. 132 (Fig. 5). This goat excreted Coxiella in milk 27 and 34 days after abortion. During the first five days, the number of goats excreting Coxiella in milk was higher in the $10^{6}$ and $10^{4}$ groups than the $10^{8}$ group; later, the number of goats which shed Coxiella in milk was higher and this excretion persisted more in the $10^{6}$ group than in the $10^{8}$ and $10^{4}$ groups. Two goats (one of the $10^{8}$ group and one of the $10^{6}$ group) shed Coxiella until the end of the experiment, 52 days after their abortion. Four goats, ( 1 of the $10^{6}$ group, and 3 of the $10^{4}$ group) presented a discontinuous excretion. There is no statistical difference between the 3 groups for the duration of milk excretion. The mean duration of excretion (comprising continuous and discontinuous shedding) was 24 days. 


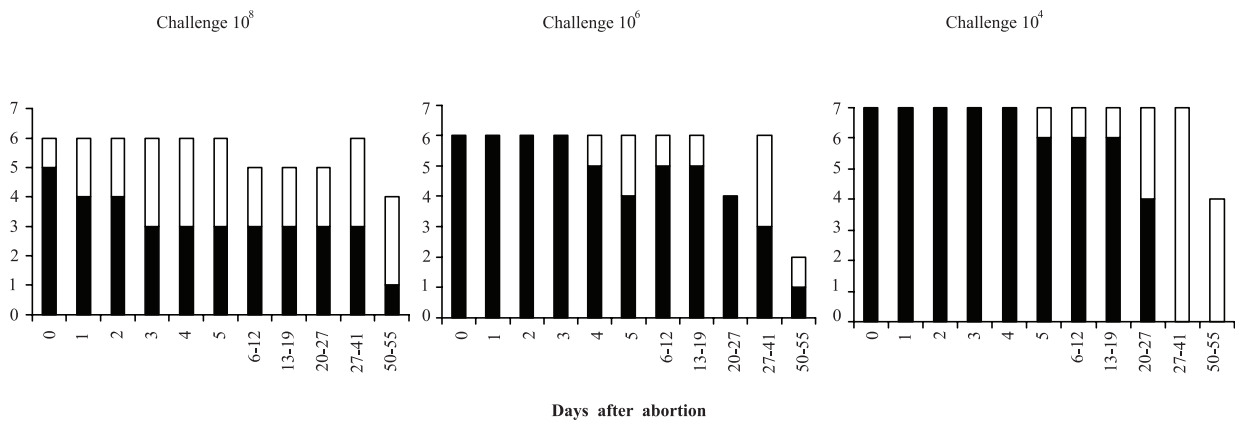

Figure 5. Assessment by PCR of the shedding of C. burnetii in milk the day of abortion and the following days. $\square$ positive PCR, $\square$ negative PCR.

\subsection{Presence of Coxiella burnetii in organs of goats after necropsy}

At the end of the experimentation, $C$. burnetii was detected in the udder of 7 goats $\left(2\right.$ of the $10^{8}$ group including goat No. 132,2 of the $10^{6}$ group and 3 of the $10^{4}$ group), and in the mammary lymph nodes of 1 goat of the $10^{4}$ group (Tab. II). No Coxiella was detected in the spleen, the liver, and the prescapular or head lymph nodes.

\section{DISCUSSION}

To study Coxiella excretion in goats, 3 doses of Coxiella were used in order to get goats with different clinical signs: one group with a majority of abortions and excretions and another group with only bacterial excretion. These doses were chosen because Brooks et al. [13] inoculated pregnant ewes at about the 100th day of gestation with $2.1 \times 10^{5}$ plaque-forming units (pfu) of phase I Nine Mile C. burnetii, and showed that only one ewe from six had aborted. The subcutaneous route of challenge is artificial and does not represent the normal routes of infection (inhalation of contaminated aerosols, and, rarely, oral route or intradermal inoculation by arthropods), but it is less dangerous than an aerosol challenge and it allowed us to get
Table II. PCR detection of $C$. burnetii from organs and mammary lymph nodes of the goat after necropsy.

\begin{tabular}{lccc}
\hline & \multicolumn{3}{c}{ Challenge dose } \\
\cline { 2 - 4 } & $10^{8}$ & $10^{6}$ & $10^{4}$ \\
\hline Time between abortion & $88 \pm 7$ & $83 \pm 0$ & $96 \pm 0$ \\
and euthanasia (days) & \multicolumn{3}{c}{} \\
PCR results (No. positive/total goats) \\
Udder & $2 / 6$ & $2 / 6$ & $3 / 7$ \\
Mammary lymph nodes & $0 / 6$ & $0 / 6$ & $1 / 7$ \\
Spleen & $0 / 6$ & $0 / 6$ & $0 / 7$ \\
Liver & $0 / 6$ & $0 / 6$ & $0 / 7$ \\
\hline
\end{tabular}

${ }^{a}$ Mean \pm SD.

inoculums under control to have reproducible results. All the subcutaneous inoculated goats, with a $C$. burnetii $\mathrm{CbC} 1$ strain aborted whatever the dose used. So, following this route of challenge, less viable bacteria of the Coxiella burnetii- $\mathrm{CbC} 1$ strain were required to induce abortion when pregnant goats were inoculated at 90 days of gestation. In this experimentation the goats were inoculated earlier than those in the experimentation of Brooks et al. [13], the counting viable bacteria were made with ID rather than pfu and the challenge strain $\mathrm{CbC} 1$ was isolated from a goat (same species in this experiment) 
rather than the Nine Mile strain isolated from a tick; this could explain the differences between the abortion rates. Any dose effect on the abortion of the goats and placental and fetal contaminations was observed here. The lower dose of Coxiella burnetii inoculated by this route seems to be sufficient to colonize placenta and fetuses, and to induce abortions. However, post-inoculation clinical signs showed a dose dependant response. Increase in temperature associated with an acute localized reaction was higher for the $10^{8}$ group compared to the $10^{4}$ group. This could be caused by the antigenic crowd which was 10000 times higher in the $10^{8}$ group than in the $10^{4}$ group. The onset of fever that occurred on days 5-7 after inoculation for 2-3 days has also been observed after intra-venous inoculation of pregnant ewes with $10^{7}$ ID 50 of $C$. burnetii [22].

One goat, No. 132 of the $10^{8}$ group was the only goat of the experiment which aborted 12 days after inoculation. This early abortion should be due to the strong temperature elevation after the inoculation of Coxiella rather than a massive infection by the bacteria. Indeed this goat presented the highest temperature elevation (average $41.3^{\circ} \mathrm{C}$ for 3 days) among the goats of the $10^{8}$ group and Coxiella were only detected by PCR in placental cotyledons but not in vaginal discharges or in fetal organs. Coxiella were found by IFA and PCR in the placenta of all goats and in $95 \%$ of the fetuses. Placental and fetal contaminations have also been reported in naturally infected goats [23, 27, 33]. The presence of Coxiella in the stomach content of fetuses indicates that the bacteria contaminate amniotic liquid; so the placenta, which can contain up to $10^{9}$ organisms/g of tissue, and the amniotic liquid are important sources of contamination of animals and the environment.

Coxiella were detected only in the udder and mammary lymph nodes when euthanasia occurred between 34 and 83 days after abortion. Contamination of the mammary lymph nodes and udder can cause chronic infection in goats, which could shed Coxiella in milk for a long period and perhaps during successive lactating periods. Here, the goats were not milked after abortion but Coxiella excretion in residual mammary secretions was still found 52 days later, at the end of the observation. These goats would perhaps shed bacteria during several months after this last sample. Indeed in some naturally infected cows, animals could continue to shed the Coxiella in their milk during successive lactating periods [12]. During this experimentation, 6 non pregnant goats were inoculated with the Coxiella $\mathrm{CbC} 1$ strain (data not shown). The day of euthanasia at 82 days p.i., C. burnetii was found in the liver of a goat inoculated with $10^{8}$ I.D. As it has been shown in the mouse, in which Coxiella were detected in the liver 2 years after inoculation [29], Coxiella was able to colonize the liver and could induce chronic infection in the goat. The presence of Coxiella in the liver could be responsible for fecal shedding by goats. Fecal excretion was displayed in pregnant and non-pregnant goats, before and after abortion, so it appears to be an important route of contamination of the environment by the spread of contaminated dung on the fields.

After abortion, all goats excreted Coxiella either in their vaginal discharges or milk and feces. Such excretion was observed on ewes and dairy cows in naturally infected flocks $[10,11,21]$ but excretion only in colostrums was observed in ewes inoculated with the Nine Mile strain of Coxiella [13]. There was no relation between the moment of abortion and the duration of excretion, but the goats, which shed bacteria in vaginal discharges for the longest period (6 to 14 days), also shed bacteria in mammary secretions for a long time, between 24 and 52 days after abortion, but fecal excretion was very different between the goats. Coxiella excretion in milk and feces was discontinuous for several goats. In fact the number of Coxiella was low in some samples of these goats 
and the negative responses could be due to the limit of detection by PCR $\left(10^{3}\right.$ bacteria/ $\mathrm{mL})$. Discontinuous excretion has also been reported for naturally infected cows where Coxiella were detected in the milk by induction of specific antibody production in mice or guinea pigs, following i.p. inoculation of milk samples [15]. No statistical differences of fecal and milk shedding were observed between the three groups and important differences were observed between goats among a same group. This lack of differences between the groups could be explained by the high sensibility of the goat to the Coxiella infection thus hiding the dose effect. In contrast, the vaginal excretion duration was shorter for the goats of the $10^{8}$ group than for the $10^{6}$ group. With regards to all the other results (duration of gestation, milk and fecal shedding...) this observation is difficult to explain but these results should be confirmed by further experiments with more animals per group and a study of this excretion in relationship with the local immune response.

Antibody responses of goats from the $10^{8}$ group began after those of the 2 other groups and then were comparable to those of the goats from the other groups. We do not know if the high antigenic mass captured the majority of the antibodies or if the high temperature elevation significantly reduced the number of Coxiella in the organism, and so delayed the immunological response. According to the threshold of positivity given by the manufacturer of the kit, $67 \%$ of the goats had a negative ELISA test when they aborted and excreted Coxiella. These results are in accordance with the results of a study by Berri et al. [11], who carried out the relationships between the shedding of Coxiella burnetii and serological responses of 34 sheep and reported that the ELISA failed to detect half of the ewes shedding $C$. burnetii through the vaginal tract. Other studies have also shown that seronegative cows could shed $C$. burnetii in their milk $[2,12]$. However, in our study, the rate of antibody against Coxiella increased from 21 days post inoculation to the end of the experiment, but the values were under the threshold of positivity of the kit the first weeks p.i.. Further experiments could be made to adjust the threshold of positivity of this test. At 80 days p.i., $91 \%$ of inoculated goats had antibodies against Coxiella, so it appears that serological tests can be used for the diagnosis of infected flocks. However, this test with the actual threshold of positivity cannot be used to identify the individual shedder that could represent a risk for public health. Indeed, Coxiella are very resistant to a drastic environment and infectious particles can be found in cheese made with raw milk or in dung and compost containing contaminated feces. Detection of Coxiella in feces or milk by PCR, for example, appears to give more accurate results and such a diagnostic technique should limit these risks of contamination. When an abortion arises in a flock, detection of Coxiella in the placenta, the fetuses' organs and vaginal secretions within 2 days after parturition by PCR and immunofluorescence tests should be privileged. Two serological tests can be made: one on the day of abortion and the other 3 weeks later to observe an elevation of antibodies against Coxiella.

This experimental infection shows that we can induce abortions and bacterial shedding due to $\mathrm{Q}$ fever in a goat model. Moreover, the $10^{4}$ Coxiella I.D. and the PCR technique can be used for the study of vaccines against $Q$ fever or antibiotic treatments and that even the rate of abortion is higher than that observed in natural flocks.

\section{ACKNOWLEDGEMENTS}

The authors are grateful to P. Bernardet, R. Delaunay, D. Gauthier and D. Musset for excellent technical assistance in the maintenance of the animals, and Agnès Moutoussamy and Karine Ladenise for laboratory technical assistance. We also appreciate the assistance rendered by Serge Bernard for statistical 
analysis. We wish to thank Laurence Guilloteau and Patricia Berthon for her help in writing the manuscript. This work was supported by DGAL (grant S98/34) and INRA.

\section{REFERENCES}

[1] Abinanti F.R., Welsh H.H., Winn J.F., Lennette E.H., Q fever studies. XIX. Presence and epidemiologic significance of Coxiella burnetii in sheep wool, Am. J. Hyg. 61 (1955) 362.

[2] Adesiyun A.A., Jagun A.G., Kwaga J.K., Tekdek L.B., Shedding of Coxiella burnetii in milk by Nigerian dairy and dual purposes cows, Int. J. Zoonoses 12 (1985) 1-5.

[3] Aitken I.D., Clinical aspects and prevention of Q fever in animals, Eur. J. Epidemiol. 5 (1989) 420-424.

[4] Babudieri B., Q fever: a zoonosis, Adv. Vet. Sci. 5 (1959) 81-154.

[5] Baca O.G., Paretsky D., Q fever and Coxiella burnetii: a model for host-parasite interactions, Microbiol. Rev. 47 (1983) 129-149.

[6] Behymer D., Riemann, H.P., Coxiella burnetii infection (Q fever), J. Am. Vet. Med. Assoc. 194 (1989) 764-767.

[7] Behymer D.E., Biberstein E.L., Riemann H.P., Franti C.E., Behymer D.E., Ruppanner R., Bushnell R., Crenshaw G., Q fever (Coxiella burnetii) investigations in dairy cattle: Challenge of immunity after vaccination, Am. J. Vet. Res. 37 (1976) 631-634.

[8] Benson W.W., Brock D.W., Mather J., Serologic analysis of a penitentiary group using raw milk from a $\mathrm{Q}$ fever infected herd, Public Health Rep. 78 (1963) 707-710.

[9] Berri M., Laroucau K., Rodolakis A., The detection of Coxiella burnetii from ovine genital swabs, milk and fecal samples by the use of a single touchdown polymerase chain reaction, Vet. Microbiol. 72 (2000) 285-293.

[10] Berri M., Souriau A., Crosby M., Crochet D., Lechopier P., Rodolakis A., Relationships between the shedding of Coxiella burnetii, clinical signs and serological responses of 34 sheep, Vet. Rec. 148 (2001) 502-505.

[11] Berri M., Souriau A., Crosby M., Rodolakis A., Shedding of Coxiella burnetii in ewes in two pregnancies following an episode of Coxiella abortion in a sheep flock, Vet. Microbiol. 85 (2002) 55-60.

[12] Biberstein E.L., Behymer D.E., Bushnell R., Crensshaw G., Riemann H.P., Franti C.E., A survey of $\mathrm{Q}$ fever (Coxiella burnetii) in
California dairy cows, Am. J. Vet. Res. 35 (1974) 1577-1582.

[13] Brooks D.L., Ermel R.W., Franti C.E., Ruppanner R., Behymer D.E., Williams J.C., Stephenson J.C., Q fever vaccination of sheep: challenge of immunity in ewes, Am. J. Vet. Res. 47 (1986) 1235-1238.

[14] Derrick E.H., Q fever, a new fever entity: clinical features, diagnosis and laboratory investigation, Med. J. Aust. 2 (1937) 281299.

[15] Durand M.P., L'excrétion lactée et placentaire de Coxiella burnetii, agent de la Fièvre $\mathrm{Q}$, chez la vache. Importance et prévention, Bull. Acad. Natl. Med. 177 (1993) 935-945.

[16] Fishbein D.B., Raoult D., A cluster of Coxiella burnetii infections associated with exposure to vaccinated goats and their unpasteurized dairy products, Am. J. Trop. Med. Hyg. 47 (1992) 35-40.

[17] Hatchette T.F., Hudson R.C., Schlech W.F., Campbell N.A., Hatchette J.E., Ratnam S., Raoult D., Marrie T.J., Donovan C., Goatassociated Q fever: a new disease in Newfoundland, Emerg. Infect. Dis. 7 (2001) 413419.

[18] Hilbink F., Penrose M., Kovacova E., Kazar J., Q fever is absent from New Zealand, Int. J. Epidemiol. 22 (1993) 945-949.

[19] Ho T., Htwe K.K., Yamasaki N., Zhang G.Q., Ogawa M., Yamaguchi T., Fukushi H., Hirai K., Isolation of Coxiella burnetii from dairy cattle and ticks, and some characterization of the isolates in Japan, Microbiol. Immunol. 39 (1995) 663-671.

[20] Literak I., Kroupa1 L., Herd-level Coxiella burnetii seroprevalence was not associated with herd-level breeding performance in Czech dairy herds, Prev. Vet. Med. 33 (1998) 261-265.

[21] Lorenz H., Jager C., Willems H., Baljer G., PCR detection of Coxiella burnetii from different clinical specimens, especially bovine milk, on the basis of DNA preparation with a silica matrix, Appl. Environ. Microbiol. 64 (1998) 4234-4237.

[22] Martinov S.P., Neikov P., Popov G.V., Experimental Q fever in sheep, Eur. J. Epidemiol. 5 (1989) 428-431.

[23] Palmer N.C., Kierstead M., Key D.W., Williams J.C., Peacock M.G., Vellend H., Placentitis and abortion in goats and sheep in Ontario caused by Coxiella burnetii, Can. Vet. J. 24 (1983) 60-61.

[24] Rady M., Glavits R., Nagy G.Y., Demonstration in Hungary of $\mathrm{Q}$ fever associated with 
abortions in cattle and sheep, Acta Vet. Hungarica 33 (1985) 169-176.

[25] Rehacek J., Epidemiology and significance of Q fever in Czechoslovakia, Zentralbl. Bakteriol. Mikrobiol. Hyg. 267 (1987) 16-19.

[26] Richardus J.H., Donkers A., Dumas A.M., Schaap G.J., Akkermans J.P., Huisman J., Valkenburg H.A., Q fever in the Netherlands: a sero-epidemiological survey among human population groups from 1968 to 1983 , Epidemiol. Infect. 98 (1987) 211-219.

[27] Sanford S.E., Josephson G.K., MacDonald A., Coxiella burnetii ( $Q$ fever) abortion storms in goat herds after attendance at an annual fair, Can. Vet. J. 35 (1994) 376-378.

[28] Stein A., Raoult D., Pigeon pneumonia in Provence: a bird-borne Q fever outbreak, Clin. Infect. Dis. 29 (1999) 617-620.

[29] Stein A., Lepidi H., Mege J.L., Marrie T.J., Raoult D., Repeated Pregnancies in BALB/c Mice Infected with Coxiella burnetii Cause Disseminated Infection, Resulting in Stillbirth and Endocarditis, J. Infect. Dis. 181 (2000) 188-194.

[30] Stoker M.G.P., Brown R.D., Kett F.J.L., Collings P.C., Marmion B.P., Q fever in Britain. Isolation of Rickettsia burneti from pla- centa and wool of sheep in an endemic area, J. Hyg. 53 (1995) 313.

[31] Tissot-Dupont H., Torres S., Nezri M., Raoult D., Hyperendemic focus of Q fever related to sheep and wind, Am. J. Epidemiol. 150 (1999) 67-74.

[32] van Moll P., Baumgartner W., Eskens U., Hanichen T., Immunocytochemical demonstration of Coxiella burnetii antigen in the fetal placenta of naturally infected sheep and cattle, J. Comp. Pathol. 109 (1993) 295-301.

[33] Waldhalm D.G., Stoenner H.G., Simmons R.E., Thomas L.A., Abortion associated with Coxiella burnetii infection in dairy goats, $\mathrm{J}$. Am. Vet. Med. Assoc. 173 (1978) 15801581.

[34] Welsh H.H., Lennette E.H., Abinanti F.R., Winn J.F., Airborne transmission of Q fever: the role of parturition in generation of infective aerosol, Ann. N. Y. Acad. Sci. 70 (1957) 528-540.

[35] Yanase T., Muramatsu Y., Inouye I., Okabayashi T., Ueno H., Morita C., Detection of Coxiella burnetii from dust in a barn housing dairy cattle, Microbiol. Immunol. 42 (1998) 51-53.

\section{To access this journal online: www.edpsciences.org}

